\title{
Adaptive Control of Truss Structures for Gossamer Spacecraft*
}

\author{
Bong-Jun Yang, Anthony J. Calise, James I. Craig, \\ School of Aerospace Engineering, Georgia Institute of Technology \\ Atlanta, GA 30332 \\ Mark S. Whorton \\ NASA Marshall Space Flight Center \\ Huntsville, AL
}

\begin{abstract}
Neural network-based adaptive control is considered for active control of a highly flexible truss structure which may be used to support solar sail membranes. The objective is to suppress unwanted vibrations in SAFE (Solar Array Flight Experiment) boom, a test-bed located at NASA. Compared to previous tests that restrained truss structures in planar motion, full three dimensional motions are tested. Experimental results illustrate the potential of adaptive control in compensating for nonlinear actuation and modeling error, and in rejecting external disturbances.
\end{abstract}

\section{INTRODUCTION}

A solar sail is an example of a gossamer structure that has been proposed as a cost effective source of space propulsion for a variety of future space exploration missions. Solar sailcraft gain momentum from reflected photons, and the continuous sunlight pressure provides sufficient propulsive energy for space missions that otherwise are only possible with conventional rocket systems using significant amounts of propellant. ${ }^{1}$ Currently, solar sail technology is being developed by the In-Space Propulsion Technology Program managed by NASA's Science Mission Directorate and implemented by the In-Space Propulsion Technology Project at Glenn Research Center. The program's objective is to develop in-space propulsion technologies that enable NASA space science missions by significantly reducing cost, mass and travel times.

In general, the momentum transferred by a single photon is extremely small, and solar sails need to span a very large area to capture and reflect photons in order to achieve a sufficient propulsive force. As a result, useful missions are only possible by use of ultra-lightweight sail films, lightweight deployable booms, and miniature avionics hardware. This makes solar sail structures unique in their constraints on mass, strength and stiffness. ${ }^{2}$ From the perspective of maneuvering/steering, solar sailcraft are large gossamer structures that tightly couple attitude and structural dynamics to achieve thrust vector control. The vehicle attitude determines the orientation of the reflective sail surface with respect to the sun and hence determines the direction of the resultant thrust vector. Structural dynamics may impact the thrust vector accuracy by inducing a jitter in the reflected solar radiation and hence affecting direction and magnitude of the thrust vector. In addition, bending vibrations of the booms and sail membranes may limit or adversely affect the stability and performance of the attitude control system.

Depending on imposed assumptions regarding the flexibility of solar sailcraft, various hardware designs and control algorithms for solar sailcraft attitude dynamics have been proposed in the literature. In [3-5], various

*This research was funded by NASA Marshall Space Flight Center, under grant number NAG8-1912. 
hardware configurations and corresponding attitude control systems are investigated in relation to mission trajectories and orbits, with a significant uncertainty being solar radiation pressure disturbance caused by an uncertain offset between the center-of-mass and the center-of-pressure. In those methods, the problem of flexibility is avoided by assuming that the slow maneuvering required for low-thrust propulsion of solar sailcraft still makes a low bandwidth attitude control system look "fast" in relative time-scales. ${ }^{2}$ In [6], a solar sailcraft is modeled as a linear flexible 6 degree-of-freedom spacecraft, and different attitude control techniques are compared in the presence of parametric uncertainties. Ref. [7] considers flexibility and its influence on control effectiveness using idealized two-dimensional models. A distributed parameter model for a flexible solar sailcraft is idealized as a rotating central hub with two opposing flexible booms, and linear feedback torque control is applied at the central hub. Another problem involves control of the solar sail film which involves control of both the dynamics of an ultra-flexible structure and the prevention/elimination of wrinkles. For this purpose distributed actuation and sensing inside the membrane of the gossamer structure have been investigated ${ }^{8-10}$

In the development of an appropriate control method, major challenges are associated with the uncertainties inherent in flexible solar sails because a comprehensive test for structural analysis is not possible in ground tests due to gravity on Earth. Even when the vacuum and thermal conditions of the space environment are well simulated, solar sail tests must employ awkward gravity offload systems to mitigate the effects of gravity. ${ }^{11}$ Further uncertainties in the material properties, test conditions, and modelling errors make it extremely difficult to obtain accurate flexibility characteristics of a flexible solar sail. Therefore, it is highly desirable for a control system to be able to adapt and compensate for system uncertainties.

In this paper, neural network (NN)-based adaptive control is considered to address the flexibilities in a truss structure that supports solar sail membranes with the objective being to suppress unwanted vibrations. The SAFE (Solar Array Flight Experiment) boom is used as a test bed for evaluating the adaptive control method. This structure was previously used in a Space Shuttle mission, and it has now been set up for control-structure interaction studies at the NASA Marshall Space Flight Center. In this study, three pairs of collocated accelerometers and air-jet thrusters mounted at the tip of the boom are utilized to suppress unwanted vibrations. The experiment is carried out by considering three-dimensional motions of the boom unlike the previous approaches that only considered planar motions of a truss structure. ${ }^{12-15}$ A critical feature that distinguishes three-dimensional motion from planar motions is the existence of coupling between bending in any two perpendicular directions. Also, as a result of long mission times, the boom may suffer gradual damage that results in inelastic deformation. For example, an inelastic bow in the boom will couple the bending and torsion at the tip where accelerometers and thrusters are mounted. In [16], it is shown that the SAFE boom severely violates Euler-Bernoulli beam model. This poses serious challenges in modelling and control design for the structure. On one hand, structural dynamics are represented using high dimension finite element models. On the other hand, control design requires model-reduction of the finite element model into a manageable state-space model while maintaining its essential features. In this paper, instead of going through this process, we illustrate a NN-based adaptive control design in which a previous linear control design approach, which performed poorly on the current structure, is augmented to account for modeling uncertainty.

The adaptive method employed in this paper is essentially the same as the method in [17]. A NN is employed to augment a linear controller and approximately cancel the effect of modeling error. It is well established that a NN can approximate any continuous function to any desired accuracy on a bounded set, ${ }^{18}$ and this has been one of the main reasons given for using a NN in adaptive control approaches. ${ }^{19-21}$ In an output feedback setting, a method that uses a memory unit of input/output delays to approximate an uncertainty has been proposed ${ }^{22}$ and shown to be effective in output feedback applications. ${ }^{23-28} \ln$ this paper, taking the path in $[25,29]$, a linear controller is designed assuming that bending in the $X-Y$ directions are decoupled from each other as well as from torsion. Two proportional-integral (PI) controllers are designed identically assuming identical modal properties in the $X-Y$ directions. This greatly simplifies the design procedure compared to designing a single controller for the coupled system of dynamics that is not available in our study. Separate but identical NNs are added to compensate for structural uncertainties. Since the PI controllers are intended to control only bending motion in a single direction and the NNs are implemented independently, the overall design is equivalent to the decentralized approach described in [17]. Using experimental tests, we illustrate that the adaptive method is effective in compensating for dead zone nonlinearities present in the thrusters as well as structural uncertainties. The experimental results are further evaluated through comparison with simulation results in which a $\mathrm{PI}$ controller fails to work because of structural uncertainties.

The paper is organized as follows. First, we describe the experimental set-up for the SAFE boom and provide modal properties obtained through preliminary tests. Next, we elaborate on how the sensors and actuators are allocated in order to carry out the design of the linear controller in the X-Y plane, how the PI controller is designed, 
and how NNs are added. Next, experimental results are presented. After simulation results are compared to the experimental results to draw conclusions for the experimental results, conclusions are given at the end.

\section{SYSTEM DESCRIPTION}

\section{TEST-BED}

Figure 1 depicts the SAFE boon, which is $30 \mathrm{~m}$ long and composed of 132 bays. The experiment is a large flexible structure which has numerous low frequency, coupled, tightly spaced lightly damped modes. ${ }^{30}$ Its current hardware includes 12 piezoelectric strain sensors, 12 accelerometers, 9 piezoelectric actuators, and 3 proportional valve air-jet thrusters. In this research, 3 collocated pairs of ADXL320 dual-axis accelerometers from Analog

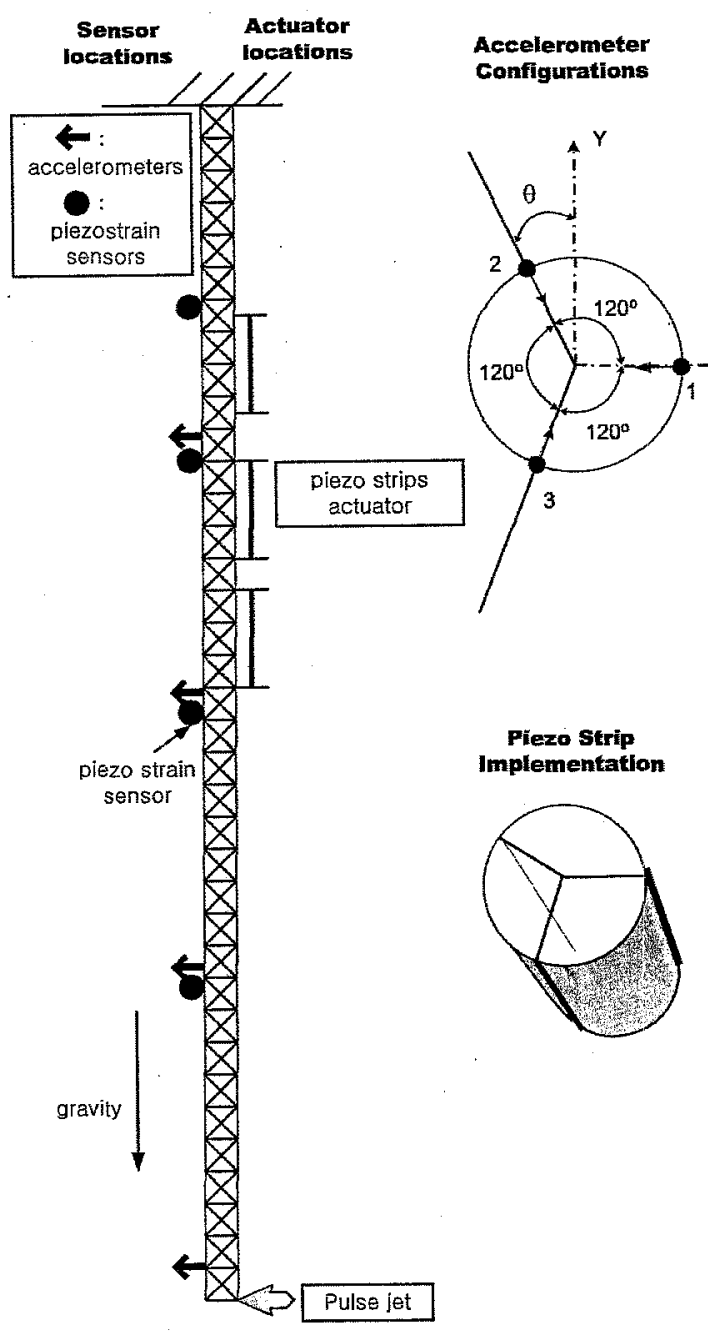

Figure 1: SAFE boom test-bed

Devices Inc. ${ }^{31}$ and EVP proportional control valve air-jet thrusters from Clippard Instrument Laboratory, Inc. ${ }^{32}$ are used for vibration suppression tests in a configuration shown on the right upper section in Figure 1 . The measured acceleration signals are processed by a National Instruments Labview routine before being used in a Labview Real-time software module which runs at $200 \mathrm{~Hz}$. The controller is imported from Matlab Simulink input-output blocks through the Simulation Interface Toolkit provided by Labview. This make it possible for Simulink blocks that are complied by Simulink Real-time Workshop to be imported as a Labview routine. The 


\begin{tabular}{|c|c|c|c|c|c|c|c|c|}
\hline & \multicolumn{2}{|c|}{ X mode } & \multicolumn{2}{c|}{$\mathrm{Y}$ mode } & \multicolumn{2}{c|}{ Torsion } & \multicolumn{2}{c|}{$\mathrm{Z}$ mode } \\
& $\omega(\mathrm{Hz})$ & $\zeta(\%)$ & $\omega(\mathrm{Hz})$ & $\zeta(\%)$ & $\omega(\mathrm{Hz})$ & $\zeta(\%)$ & $\omega(\mathrm{Hz})$ & $\zeta(\%)$ \\
\hline 1 & 0.182 & 1.43 & 0.185 & 2.64 & 2.060 & 1.7 & 15.861 & 0.3 \\
\hline 2 & 0.787 & 0.93 & 0.823 & 0.91 & $\cdot$ & $\cdot$ & $\cdot$ & $\cdot$ \\
\hline 3 & 2.020 & 5.86 & 2.141 & 7.62 & $\cdot$ & $\cdot$ & $\cdot$ & $\cdot$ \\
\hline 4 & 4.061 & 6.69 & 4.241 & 7.03 & $\cdot$ & $\cdot$ & $\cdot$ & $\cdot$ \\
\hline
\end{tabular}

Table 1: Modal Coefficients

control signals from the Labview embedded controller software are converted to $2.5 \mathrm{kHz}$ pulse-width modulation (PWM) signal in Labview to drive the EVP valves. As a result, a command from 0 to 1 corresponds to PWM from 0 to $100 \%$. The air begins to flow at approximately 30\% PWM signal, and the valve is fully open at $100 \%$ PWM signal. ${ }^{32}$ Ignoring the PWM block and assuming that the 0-1 signal is proportional to the air jet flow (liters/minute), the actuator can be characterized as shown in Figure 2 where $u_{i}(i=1,2,3)$ is the dimensionless control signal to the Labview PWM routine driving each valve. Note that this actuator presents both a dead zone and a saturation nonlinearity.



Figure 2: Actuator Characteristic

\section{STRUCTURAL PROPERTIES}

Table 1 shows modal properties obtained by modal analysis of impact data up to 4 bending modes, the first torsional mode, and the first axial. mode. In the impact testing, it was observed that $X-Y$ motions are not decoupled, and the modal coefficients in Table 1 are mainly used as a guideline for the design of a linear controller for providing damping to the structure. In performing simulations, these properties are modified to match the experimental data to construct a simulation model.

\section{CONTROL DESIGN}

Figure 3 shows the adaptive control architecture in which three thrusters and three accelerometers are treated as the actuators and sensors. Two independent controllers are designed to suppress vibrations in the $X$ and $Y$ direction, which belongs to the class of decentralized controllers treated in [17].

\section{OUTPUT AND CONTROL ALLOCATION}

Figure 4(a) depicts how sensors and actuators are located at the tip of the SAFE boom. Each accelerometer 


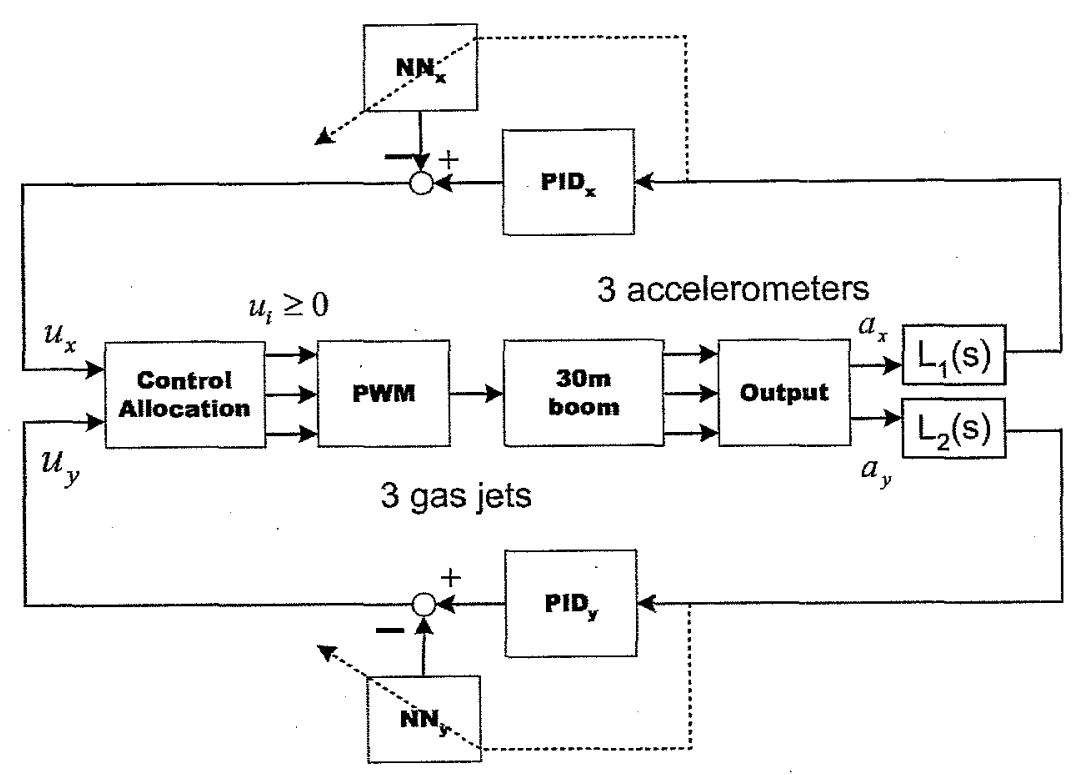

Figure 3: Control Architecture

measures accelerations in radial directions and tangential directions. The inertial acceleration $\vec{a}=a_{x} i+a_{y} \boldsymbol{j}(\boldsymbol{i}, \boldsymbol{j}$ are unit vectors in $X-Y$ coordinates) at the origin $O$ in Figure 4(a) results in the following accelerometer readings in the radial directions:

$$
\left[\begin{array}{l}
a_{1} \\
a_{2} \\
a_{3}
\end{array}\right]=\left[\begin{array}{ll}
-\cos \phi_{1} & -\sin \phi_{1} \\
-\cos \phi_{2} & -\sin \phi_{2} \\
-\cos \phi_{3} & -\sin \phi_{3}
\end{array}\right]\left[\begin{array}{l}
a_{x} \\
a_{y}
\end{array}\right]=\underbrace{\left[\begin{array}{cc}
-1 & 0 \\
\sin \theta & -\cos \theta \\
\sin \theta & \cos \theta
\end{array}\right]}_{T}\left[\begin{array}{l}
a_{x} \\
a_{y}
\end{array}\right],
$$

where $\phi_{1}=0, \phi_{2}=120^{\circ}, \phi_{3}=240^{\circ}, \theta=30^{\circ}$. Conversely, the accelerations $a_{x}$ and $a_{y}$ in Figure 3 can be derived from acceleration readings $a_{i}(i=1,2,3)$ as follows:

$$
\left[\begin{array}{l}
a_{x} \\
a_{y}
\end{array}\right]=\left(T^{\top} T\right)^{-1} T^{\top}\left[\begin{array}{l}
a_{1} \\
a_{2} \\
a_{3}
\end{array}\right]=\frac{2}{3}\left[\begin{array}{ccc}
-1 & \sin \theta & \sin \theta \\
0 & -\cos \theta & \cos \theta
\end{array}\right]\left[\begin{array}{l}
a_{1} \\
a_{2} \\
a_{3}
\end{array}\right] .
$$

In the same manner, the control signals $u_{x}$ and $u_{y}$ in Figure 3 should be realized using single directional control signals $u_{i} \geq 0(i=1,2,3)$ that are fed to the PWM routine in Figure 3 . That is, the $u_{i}$ 's are determined from $u=u_{x} i+u_{y} j=u_{1} r_{1}+u_{2} r_{2}+u_{3} r_{3}$, where $r_{i}$ is a unit vector aligned with the thrust axis of the $i$ th thruster. This leads to

$$
\left[\begin{array}{l}
u_{x} \\
u_{y}
\end{array}\right]=\left[\begin{array}{ccc}
-1 & \sin \theta & \sin \theta \\
0 & -\cos \theta & \cos \theta
\end{array}\right]\left[\begin{array}{l}
u_{1} \\
u_{2} \\
u_{3}
\end{array}\right]=T^{\top}\left[\begin{array}{l}
u_{1} \\
u_{2} \\
u_{3}
\end{array}\right] .
$$

Nonnegative solutions for each $u_{i}$ are determined as follows. After a region to which $u$ belongs is determined, $u$ is realized using two nonnegative components. For example, Figure 4(b) illustrates how $u \in \Omega_{1}$ can be realized by $u_{2}$ and $u_{3}$. A region to which $u$ belongs is decided using a parameter $R=\operatorname{atan} 2\left(u_{y}, u_{x}\right)$. As a result, control signals $u_{i}$ are allocated as follows.

1. $u \in \Omega_{1}\left(-\frac{\pi}{3} \leq R \leq \frac{\pi}{3}\right)$ $u_{1}=0$, and $u_{2}, u_{3}$ are determined by $\left[\begin{array}{l}u_{x} \\ u_{y}\end{array}\right]=\left[\begin{array}{cc}\sin \theta & \sin \theta \\ -\cos \theta & \cos \theta\end{array}\right]\left[\begin{array}{l}u_{2} \\ u_{3}\end{array}\right]$.

2. $u \in \Omega_{2}\left(\frac{\pi}{3}<R \leq \pi\right)$ $u_{2}=0$, and $u_{1}, u_{3}$ are determined by $\left[\begin{array}{l}u_{x} \\ u_{y}\end{array}\right]=\left[\begin{array}{cc}-1 & \sin \theta \\ 0 & \cos \theta\end{array}\right]\left[\begin{array}{l}u_{1} \\ u_{3}\end{array}\right]$. 


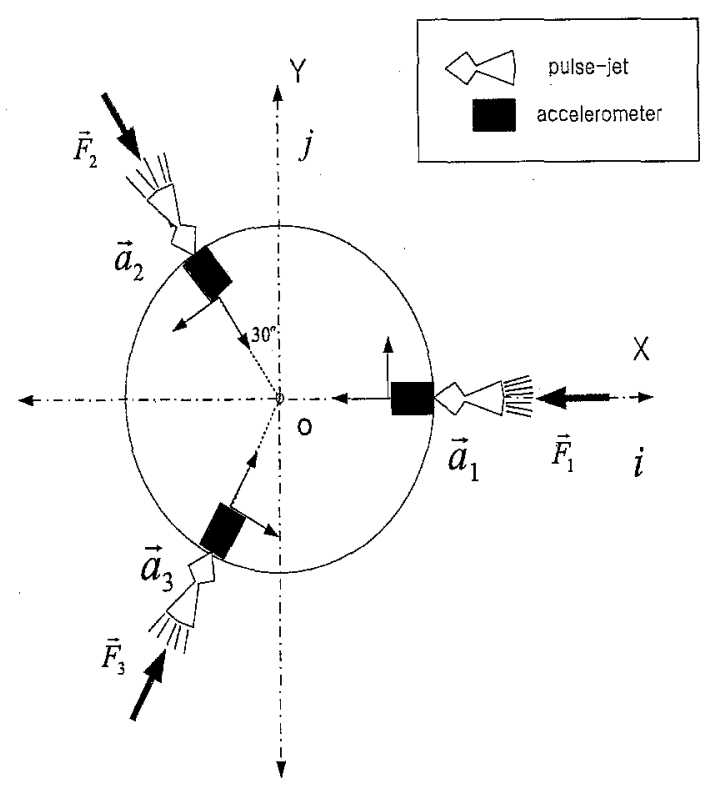

(a) Configuration for accelerometers and air-jet thrusters

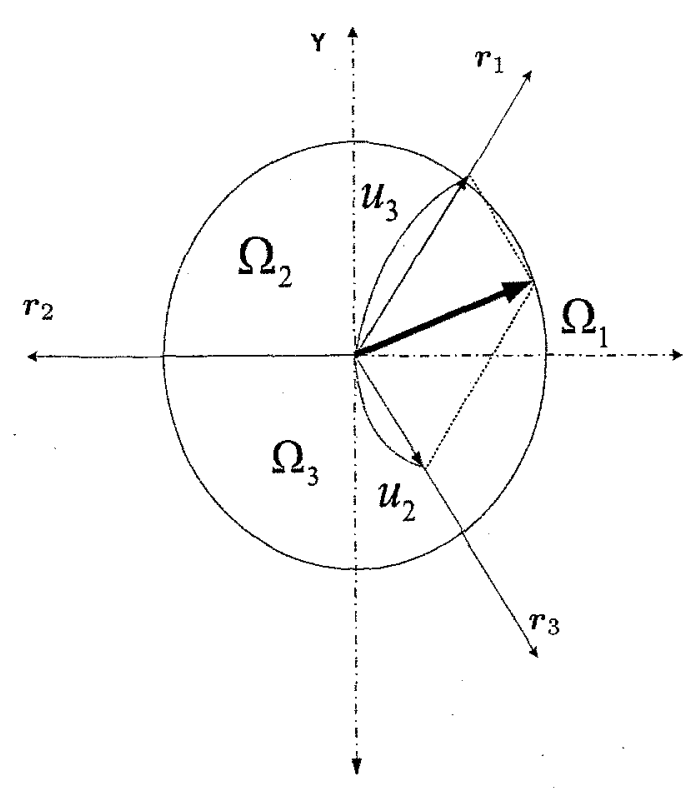

(b) Control Allocation

Figure 4: Outputs and Control Allocation

3. $u \in \Omega_{3}\left(-\pi \leq R<-\frac{\pi}{3}\right)$

$u_{3}=0$, and $u_{1}, u_{2}$ are determined by $\left[\begin{array}{l}u_{x} \\ u_{y}\end{array}\right]=\left[\begin{array}{cc}-1 & \sin \theta \\ 0 & -\cos \theta\end{array}\right]\left[\begin{array}{l}u_{1} \\ u_{2}\end{array}\right]$.

\section{LINEAR CONTROLLERS}

Since we are interested in frequencies below $3 \mathrm{~Hz}$, the acceleration signals are filtered by low-pass filters $L_{i}(s)$ $(i=1,2)$ as shown in Figure 3 , where

$$
L_{i}(s)=\frac{1}{s / \omega_{l}+1}, \omega_{l}=6(H z)=12 \pi(\mathrm{rad} / \mathrm{sec}), i=1,2
$$

PI controllers are designed under the assumption that $X-Y$ dynamics are decoupled. Since Table 1 only provides modal properties of the structure, developing a high-fidelity structural model that includes actuator nonlinearities is a time-consuming procedure. Instead of attempting an elaborate model based control design, all the nonlinearities are neglected, and the following simplified structural model is used for control design

$$
\begin{aligned}
& \ddot{q}_{x_{i}}+2 \zeta_{x_{i}} \omega_{x_{i}} \dot{q}_{x_{i}}+\omega_{x_{i}}^{2} q_{x_{i}}+\phi_{x_{i}}(s)=b_{x_{i}} u_{x} \\
& \ddot{q}_{y_{i}}+2 \zeta_{y_{i}} \omega_{y_{i}} \dot{q}_{y_{i}}+\omega_{y_{i}}^{2} q_{y_{i}}+\phi_{y_{i}}(s)=b_{y_{i}} u_{y}, \quad i=1,2, \\
& a_{x}=K_{s} \ddot{x} \\
& a_{y}=K_{s} \ddot{y}
\end{aligned}
$$

where $q_{x_{i}}$ and $q_{y_{i}}$ represent modal displacements, the displacement in the $\mathrm{X}-\mathrm{Y}$ directions are given by $x=q_{x_{1}}+q_{x_{2}}$, $y=q_{y_{1}}+q_{y_{2}}$, and $s=[x, y, \dot{x}, \dot{y}]^{\top}$. The variables $a_{x}$ and $a_{y}$ are accelerations in the $\mathrm{X}$ and $Y$ directions, and the corresponding modal coefficients are given in Table 1 . The parameter values: $b_{x_{1}}=0.9, b_{x_{2}}=0.1, b_{y_{1}}=0.7$, $b_{y_{2}}=0.3, K_{s}=0.034$ were selected to match the experimental data from open-loop response tests. The terms $\phi_{x_{i}}(s)$ and $\phi_{y_{i}}(s)$ are lumped uncertainties that represent the effect of modeling error. If $\phi_{x_{i}}(s)=\phi_{y_{i}}(s)=0$, the transfer function for the $\mathrm{X}$-direction is given by

$$
\frac{a_{x}(s)}{u_{x}(s)}:=G_{x}(s)=K_{x} \frac{s^{2}\left(s^{2}+2 \zeta_{z} \omega_{z} s+\omega_{z}^{2}\right)}{\left(s^{2}+2 \zeta_{1} \omega_{1} s+\omega_{1}^{2}\right)\left(s^{2}+2 \zeta_{2} \omega_{2} s+\omega_{2}^{2}\right)},
$$


where $K_{x}=0.034, \omega_{z}=4.66 \mathrm{rad} / \mathrm{s}(=0.7417 \mathrm{~Hz}), \zeta_{z}=0.009, \omega_{1}=1.1435 \mathrm{rad} / \mathrm{s}(=0.182 \mathrm{~Hz}), \omega_{2}=$ $4.9449 \mathrm{rad} / \mathrm{s}(=0.787 \mathrm{~Hz}), \zeta_{1}=0.0143, \zeta_{2}=0.0093$. A PI controller is designed based on the transfer function in (6) using MATLAB SISO tool. Figure 5 shows the root locus plot and loop gain bode plot when the PI controller is given by

$$
G_{c}(s)=K_{p} \frac{s+z_{c}}{s},
$$

where $K_{p}=2, z_{c}=-0.5$. The closed loop poles are located at $-40.2,0,-0.050 \pm 4.89 j,-0.0306 \pm 1.1 j$. The
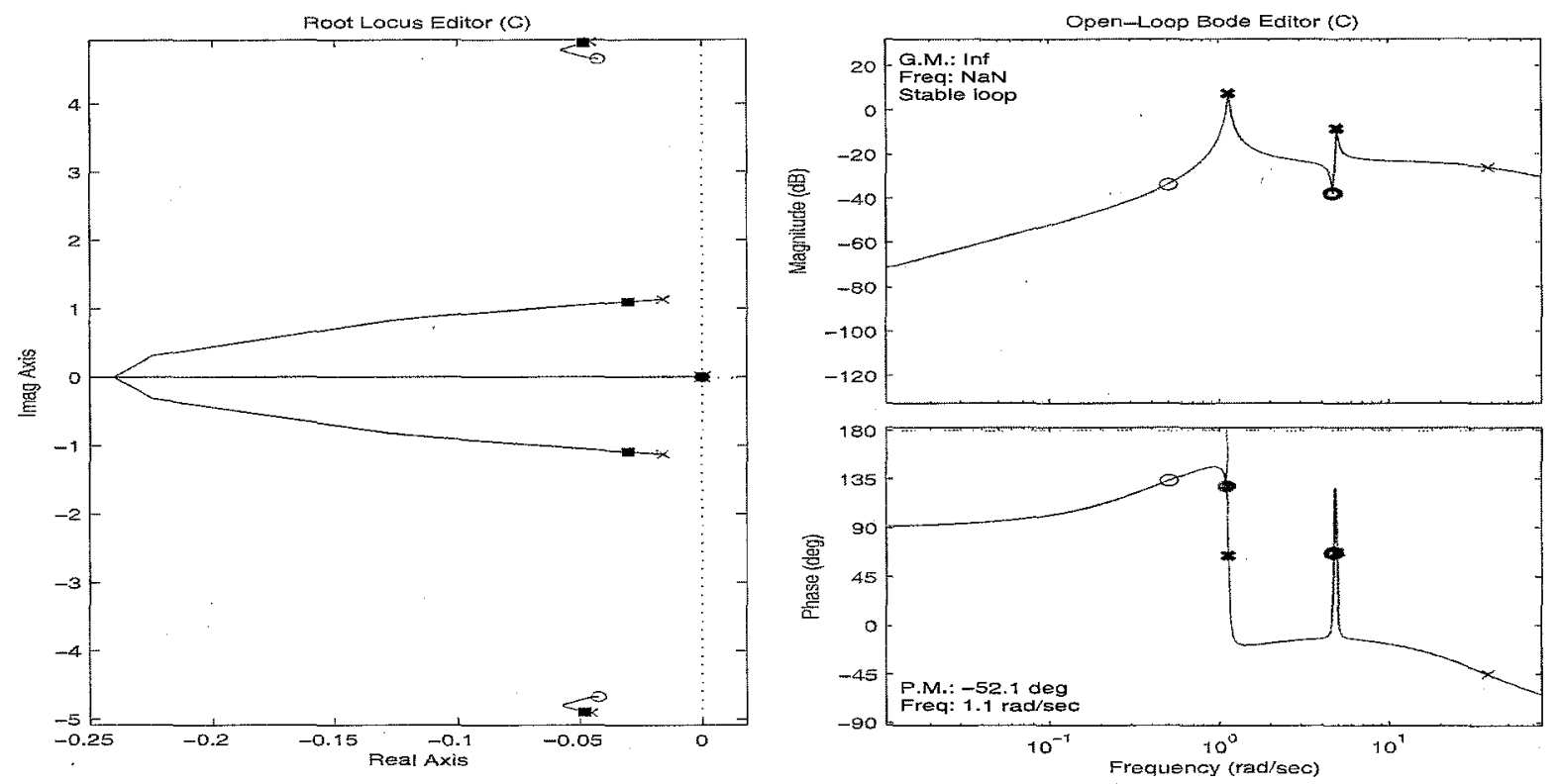

Figure 5: root locus and loop gain plots when the transfer function in (6) is regulated by the PI controller in (7)

damping ratio has increased from 0.0143 to 0.0279 while the natural frequency has slightly decreased from 1.1435 $\mathrm{rad} / \mathrm{s}$ to $1.10 \mathrm{rad} / \mathrm{s}$ for the first mode. The second mode has a damping ratio of 0.010 and natural frequency of $4.89 \mathrm{rad} / \mathrm{s}$. Therefore, the $\mathrm{PI}$ controller mainly adds damping to the first mode and has little influence on the second mode. The same controller is used in the $Y$ direction.

\section{ADAPTIVE CONTROL}

Two single hidden-layer NNs (SHLNNs) are used to compensate for uncertainties in the system dynamics as shown in Figure 3. The design approach follows the method in [26] in which an adaptive design approach for a relative degree one nonminimum phase system is presented with acceleration as a regulated variable. Since adaptive controllers are augmented identically for both $\mathrm{X}$ and $\mathrm{Y}$ directions, subscript $x$ and $y$ are dropped for simplicity of presentation in what follows.

Since the acceleration in (6) has relative degree zero with respect to the control input $u_{x}$, a filtered acceleration is chosen as the regulated output,

$$
a_{f}=L_{1}(s) a_{x}
$$

which has relative degree one. From this point, the method is identical to that in [26]. With $a_{f}$ as the regulated output, the systems in (5) and (8) can be put in a normal form whose first line becomes

$$
\dot{a}_{f}=-w_{l} a_{f}-w_{l} K_{s} \sum_{i=1}^{2}\left(2 \zeta_{i} w_{i} \dot{q}_{i}+\omega_{i}^{2} q_{i}\right)+w_{l} K_{s}(u+\Delta(s)),
$$

where the matched uncertainty $\Delta(s)$ is given by

$$
\left.\Delta(s)=-\sum_{i=1}^{2} \phi_{i}(s)\right)
$$


Let

$$
u=u_{l c}-u_{a d},
$$

where $u_{l c}$ is the $\mathrm{PI}$ control signal, and $u_{a d}$ is the adaptive signal to compensate for the uncertainty. Since the acceleration is regulated to zero, the reference model in [26] is simply zero, and the tracking error is given by

$$
e=0-a_{f}=-a_{f}
$$

A SHLNN is used to approximate the matched uncertainty $\Delta(s)$ in (10) using a memory unit of sampled input/output pairs. Following the NN approximation property in [22], the matched uncertainty is parameterized

$$
\Delta(\boldsymbol{\eta})=\boldsymbol{W}^{\top} \sigma\left(V^{\top} \eta\right)+\varepsilon(\boldsymbol{\eta}),|\varepsilon(\boldsymbol{\eta})| \leq \epsilon^{*},
$$

on a compact domain of interest under the assumption that the system dynamics are observable with respect to the regulated output. The term $\varepsilon(\boldsymbol{\eta})$ is the NN reconstruction error, which is upper bounded by $\epsilon^{*}$ on the approximation domain, and $\eta$ is the network input vector

$$
\begin{aligned}
\eta(t) & =\left[\begin{array}{lll}
1 & \bar{u}_{d}^{\top}(t) & \overline{\boldsymbol{y}}_{d}^{\top}(t)
\end{array}\right]^{\top}, \\
\overline{\boldsymbol{u}}_{d}^{\top}(t) & =\left[\begin{array}{lll}
u(t) & u(\dot{t}-d) & \cdots u\left(t-\left(n_{1}-r-1\right) d\right)
\end{array}\right]^{\top}, \\
\overline{\boldsymbol{y}}_{d}^{\top}(t) & =\left[\begin{array}{lll}
a_{f}(t) & a_{f}(t-d) \cdots a_{f}\left(t-\left(n_{1}-1\right) d\right)
\end{array}\right]^{\top},
\end{aligned}
$$

in which $n_{1}$ is the length of the window and is generally required to be greater than or equal to the system dimension, $d>0$ is a time-delay, $r$ is the relative degree of the output (one for $a_{f}$ ), $\sigma$ is a vector of squashing functions, $\sigma(\cdot)$, whose $i^{t h}$ element is defined as $\left[\sigma\left(V^{\top} \eta\right)\right]_{i}=\sigma\left[\left(V^{\top} \eta\right)_{i}\right]$. The squashing functions are chosen as sigmoidal functions

$$
\left[\sigma\left(V^{\top} \eta\right)\right]_{i}=\frac{1}{1+e^{-a\left(V^{\top} \eta\right)}}, i=1, \ldots, N
$$

where $a=1$ represents the activation potential, and $N$ is the number of neurons in the hidden layer.

The adaptive signal $u_{a d}$ is designed as

$$
u_{a d}=\widehat{\boldsymbol{W}}(t)^{\top} \sigma\left(\widehat{V}(t)^{\top} \eta\right)
$$

where $\widehat{\boldsymbol{W}}(t)$ and $\widehat{V}(t)$ are estimates for the ideal weights that are adapted on-line. The update law for $\widehat{\boldsymbol{W}}(t)$ and $\widehat{V}(t)$ follows from that in [26]

$$
\begin{aligned}
\ddot{\widehat{W}} & =-\Gamma_{W}\left[\left(\hat{\sigma}-\hat{\sigma}^{\prime} \widehat{V}^{\top} \eta\right) e+k_{\sigma} \widehat{W}\right] \\
\dot{\hat{V}} & =-\Gamma_{V}\left[e \eta \widehat{W}^{\top} \hat{\sigma}^{\prime}+k_{\sigma} \widehat{V}\right],
\end{aligned}
$$

in which $\Gamma_{W}, \Gamma_{V}>0$ are positive definite adaptation gain matrices, $k_{\sigma}>0$ is a $\sigma$-modification constant, $\hat{\sigma} \triangleq \sigma(\hat{V} \eta), \hat{\sigma}^{\prime}$ is the Jacobian computed at the estimates.

In implementation, the SHLNN consists of 5 neurons $(N=5)$, and 8 delayed values of the output, together with 7 delayed inputs, are used to construct the NN input $\eta\left(n_{1}=8\right.$ and $\left.r=1\right)$. The time delay $d=0.01$ sec., and the parameters for the $N N$ are

$$
\Gamma_{W}=1 I, \Gamma_{V}=1 I, k_{\sigma}=1
$$

where $I$ is the identity matrix with compatible dimension.

\section{EXPERIMENTAL RESULTS}

The experiment was performed by applying three cycles of a $0.17 \mathrm{~Hz}$ sinusoidal disturbance voltage to the thruster located at the tip in the X-direction. After the disturbance, the control thrusters are activated at 20 sec. When the PI controller in (7) is implemented, the closed-loop system immediately went unstable with further tweaking gains for $K_{p}$ and $K_{i}$ not being able to recover stability. Selecting $K_{i}=0$ and $K_{p}=1$ results in the stable system, whose acceleration responses are shown in Figure 6 . Notice that the disturbance in the $X$-direction leads to an acceleration in Y-direction due to structural coupling as shown in Figure $6(b)$. Moreover, the proportional 

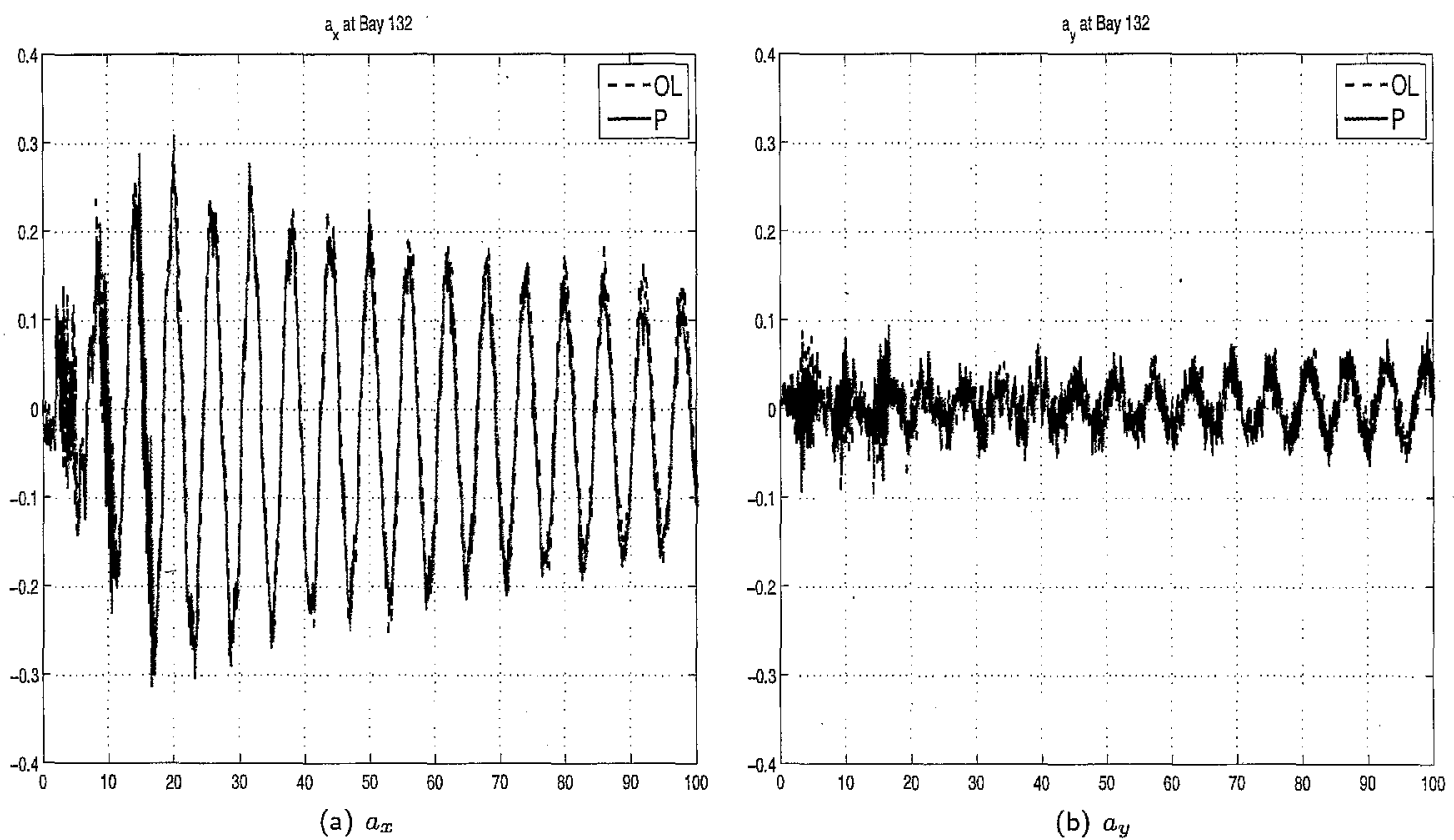

Figure 6: Acceleration responses to the initial disturbances

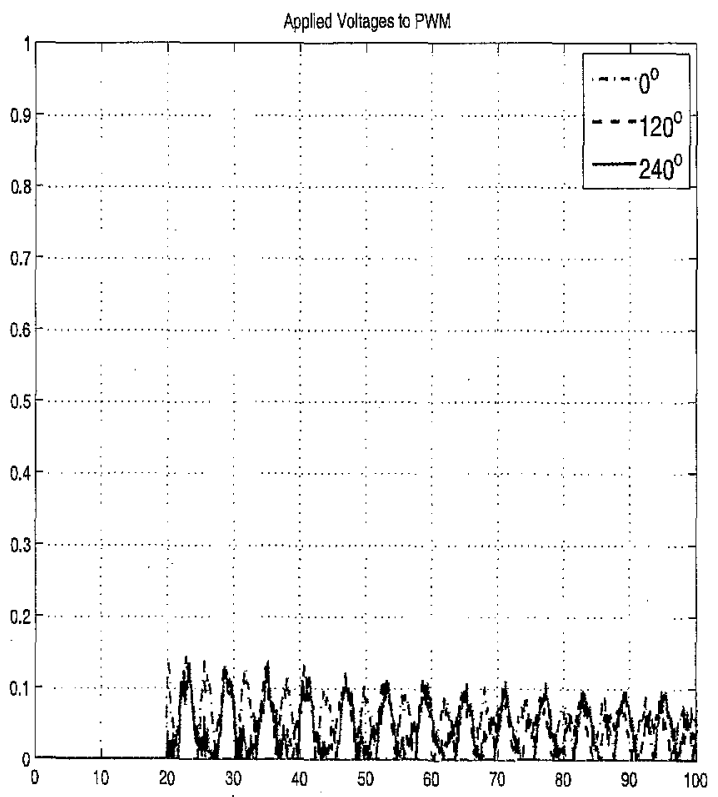

(a) $u_{i}$ for the $\mathrm{P}$ controller

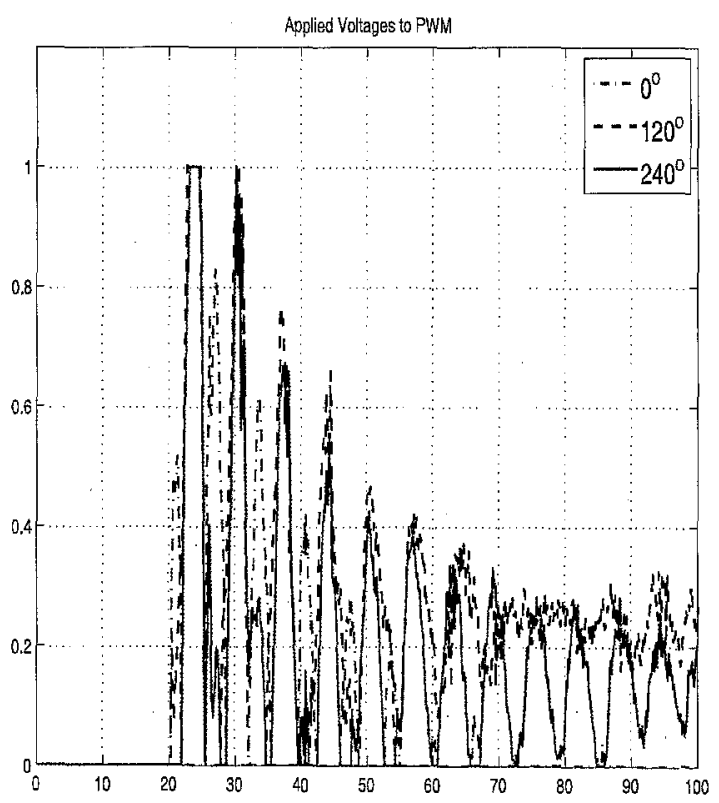

(b) $u_{i}$ for the $\mathrm{P}$ controller with NN augmented

Figure 7: Applied Control Voltages to PWM 

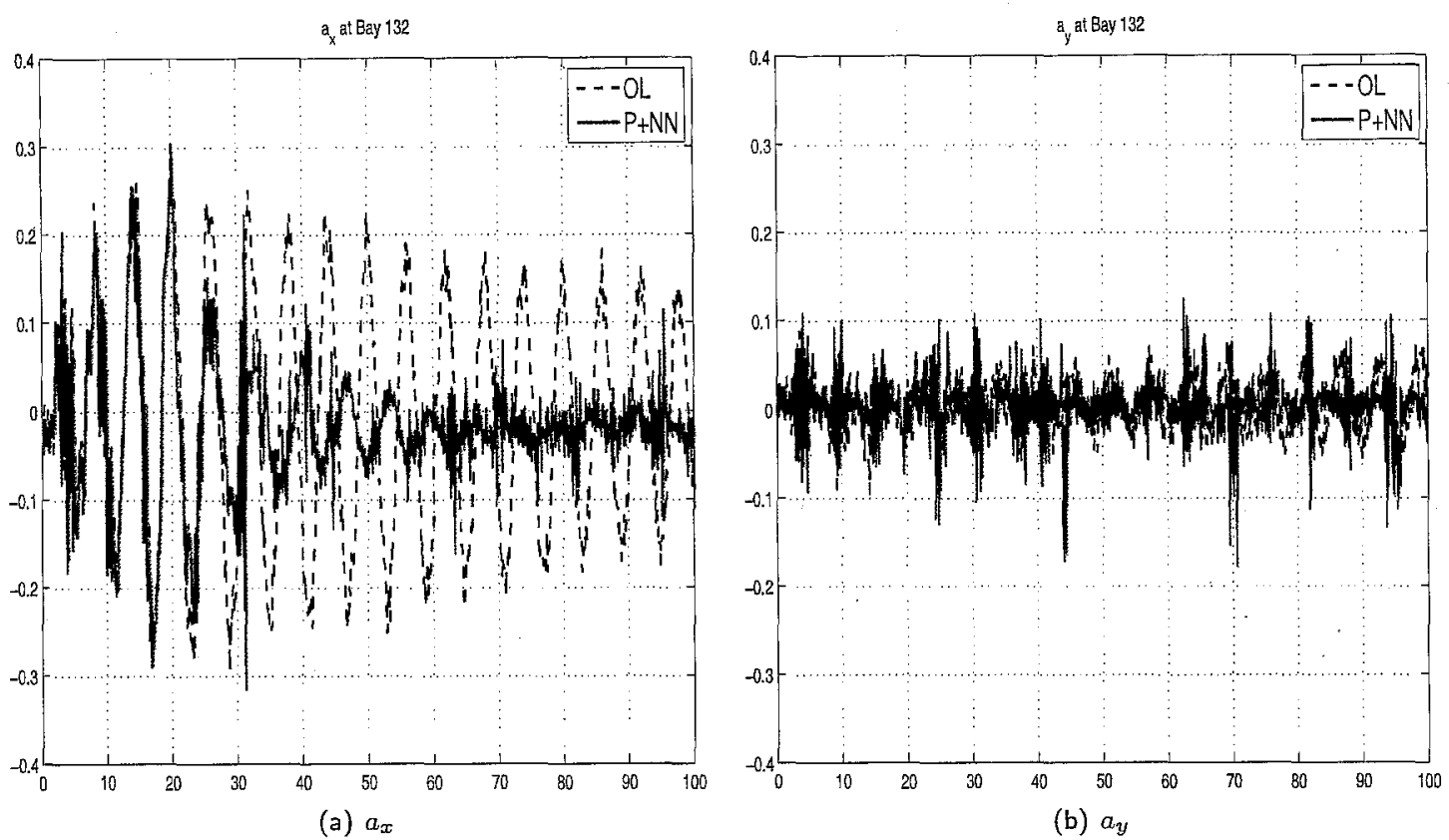

Figure 8: Comparison of acceleration responses of the open-loop to those with the $\mathrm{P}$ controller augmented by the INN

(P) controller results in almost identical responses to those of the open-loop system, implying that the proportional controller is not effective in suppressing vibrations. Figure 7 (a) reveals that the control voltages applied to PWM never exceeded the dead zone value, and the $\mathrm{P}$ controller essentially resulted in open-loop responses.

Whereas a stabilizing combination for $K_{p}$ and $K_{i}$ was not found, generally proportional velocity feedback should be able to provide damping. ${ }^{33}$ However, since a velocity sensor is not available, this can only be approximated by pure integral feedback. When a pure integral controller $\left(K_{p}=0\right.$ and $\left.K_{i}=0.2\right)$ was implemented, the accelerometer bias caused the integrator to wind up and the structure was displaced to a point in which the thrusters with fully open valves are in balance with the recovering force due to structural stiffness.

Figure 8 shows the acceleration responses when the $P$ controller is augmented by the NN ("P+NN"). The vibrations in $X$-direction are well suppressed by the thrusters, and the accelerations in the $X-Y$ directions subsides to the same level. Figure 7 (b) shows that with the NN augmented, the dead zone nonlinearity is overcome by the adaptive signal. Comparing Figure $7(\mathrm{~b})$ to Figure $8(\mathrm{~b})$ reveals that spikes in $a_{y}$ occur when a pair of thrusters are switched into a different pair according to the control allocation method. This implies that non-perfect synchronization in the thrusters caused the spikes. Acceleration responses and control voltages are shown in Figures 9 and 10 for the case where a continuous sinusoidal disturbance is applied in the X direction. Figure 10(a) reveals that with the $P$ controller the control signal does not exceed the actuator dead zone and the responses in Figure 9(a) are essentially the same as the open-loop responses which exhibit resonant behavior. With the P controller augmented by the NN, Figure 9 (b) shows that the external disturbances are well attenuated.

\section{UNCERTAINTY MODELING}

In this section, we further investigate, 1) the possibility of using a washed out integrator (an integrator with its pole shifted slightly into the left hand plane), 2) the significance of actuator dead zone, and 3) the effect of structural uncertainty. 


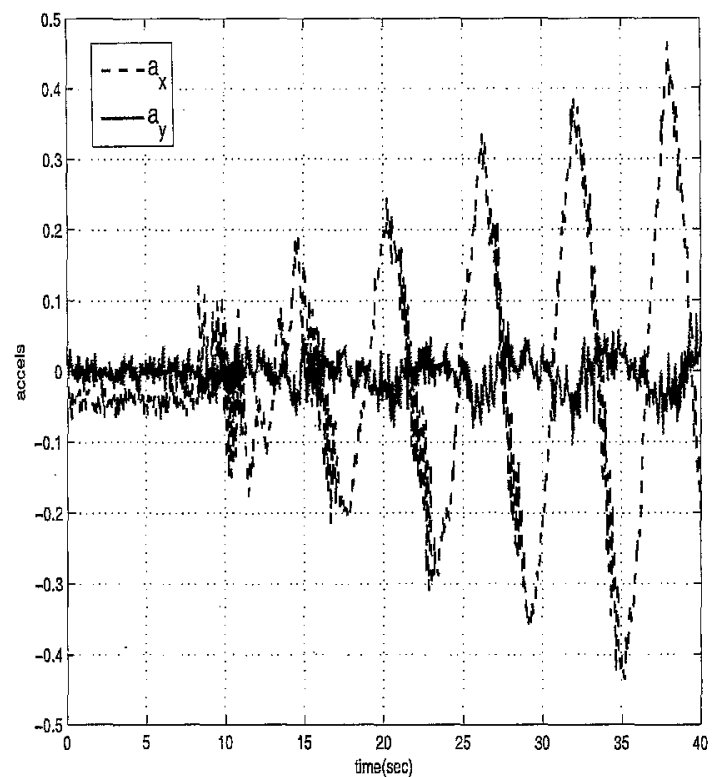

(a) $a_{x}$ and $a_{y}$ without NN

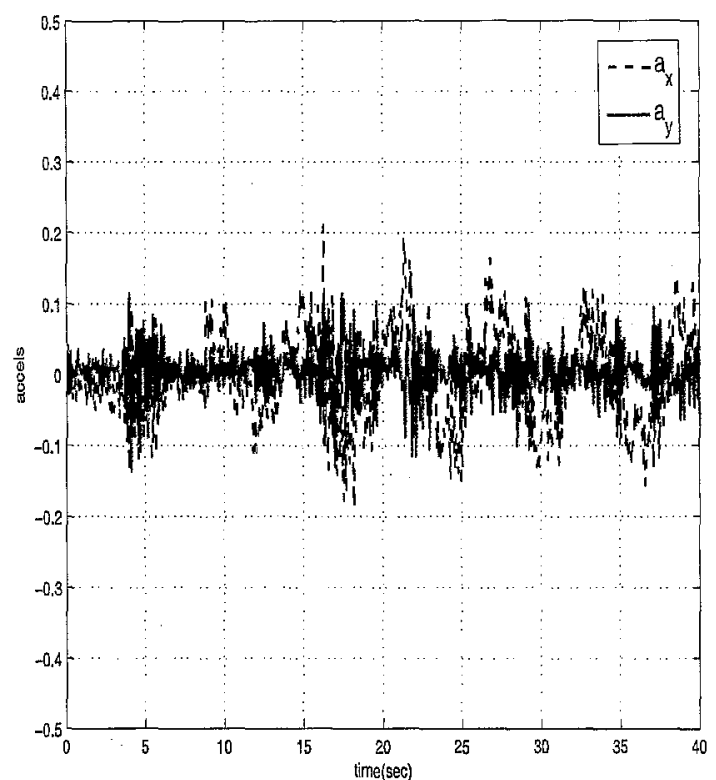

(b) $a_{x}$ and $a_{y}$ with the NN

Figure 9: Acceleration responses to continual external disturbances

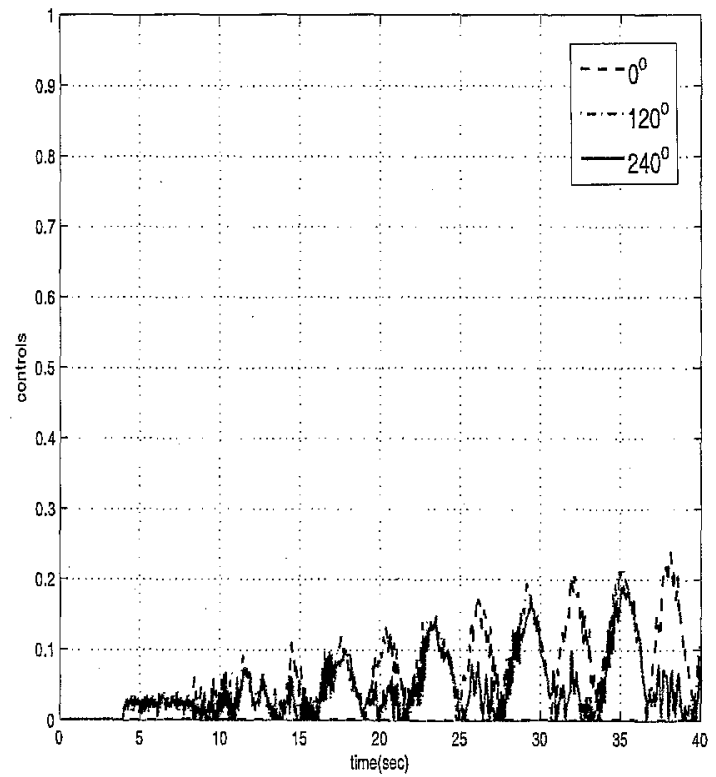

(a) $u_{i}$ without the NN



(b) $u_{i}$ with the NN

Figure 10: Control signals with continuous external disturbances 


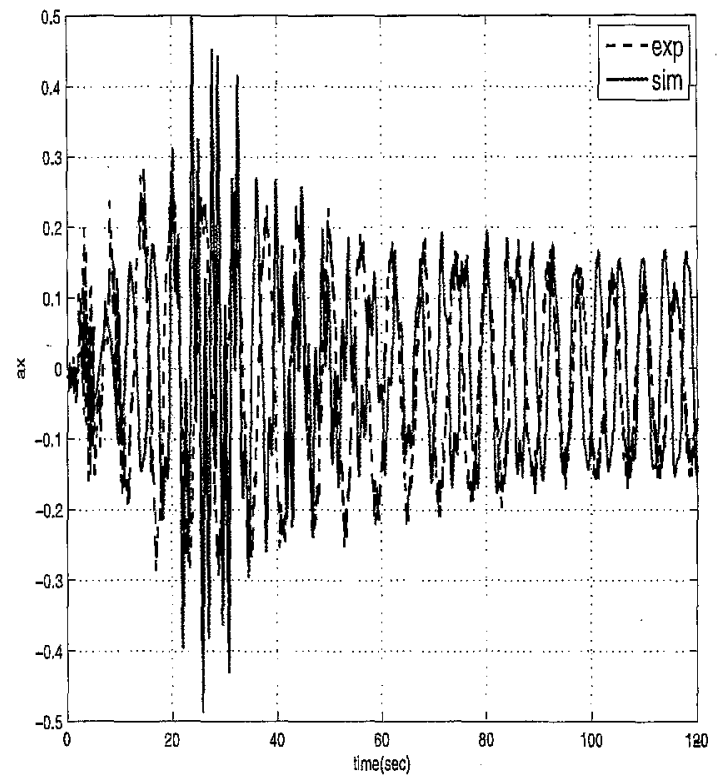

(a) $a_{x}$

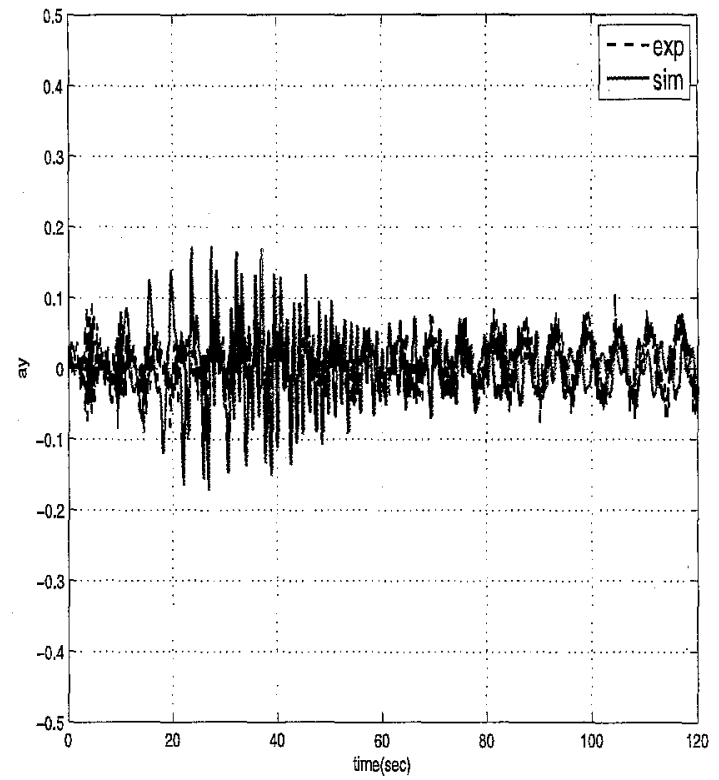

(b) $u_{i}$ with the NN

Figure 11: Comparison of Simulation model and the experimental data

\section{SIMULATION MODEL}

The linear model based on modal coefficients in Table 1 was used to design the PI controller in (7) $\left(\phi_{x_{i}}=\right.$ $\left.\phi_{y_{i}}=0\right)$. In this section specific choices for the terms $\phi_{x_{i}}, \phi_{y_{i}}$ are introduced to approximate the $X-Y$ coupling accelerations observed experimentally:

$$
\begin{aligned}
& \phi_{x_{1}}(x, y)=-\alpha_{x_{1}} x^{3}-\beta_{y_{1}}\left(y^{2}+0.1 y^{3}\right), \phi_{x_{2}}(x, y)=-\alpha_{x_{2}} x^{3}-\beta_{y_{2}}\left(y^{2}+0.1 y^{3}\right) \\
& \phi_{y_{1}}(x, y)=-\alpha_{y_{1}} y^{3}-\beta_{y_{1}}\left(x^{2}+0.1 x^{3}\right), \phi_{y_{2}}(x, y)=-\alpha_{y_{2}} y^{3}-\beta_{y_{2}}\left(x^{2}+0.1 x^{3}\right),
\end{aligned}
$$

where $\alpha_{x_{1}}=0.3, \alpha_{x_{2}}=0.1, \alpha_{y_{1}}=0.2, \alpha_{y_{2}}=0.1, \beta_{x_{1}}=\beta_{x_{2}}=\beta_{y_{1}}=\beta_{y_{2}}=0.5$. These terms are derived considering a coupled mass-spring motion in the $X$ and $Y$ directions, and they do no affect the linearized dynamics. Actuation nonlinearity is also introduced in (5) using

$$
\left[\begin{array}{l}
u_{x} \\
u_{y}
\end{array}\right]=T^{\top}\left[\begin{array}{l}
g\left(u_{1}\right) \\
g\left(u_{2}\right) \\
g\left(u_{3}\right)
\end{array}\right],
$$

where the function $g$ is depicted in Figure 2. With the nonlinearities in (19) and (20), when the system in (5) is subjected to the same disturbance used to obtain the results in Figure 6, the simulated response produced a smaller settling time. Therefore, to match the experimental data, the damping coefficient for the first mode in $\mathrm{X}$-direction was reduced to $0.3 \%$. Figure 11 compares the resulting simulation responses to the experimental data. While Figure 11(a) shows that the frequency and the damping for the first mode matches those of the experimental data, the acceleration $a_{y}$ in Figure 11(b) does not agree that well. This is due to the fact that the accelerations in the $Y$-direction stem from the unknown structural coupling, and the coupling nonlinearities in (5) are artificially devised. Nevertheless, it was felt that the match between the experimental and simulated accelerations in the $Y$ axis are adequate for a qualitative analysis.

\section{A PI CONTROLLER WITH A WASHED OUT INTEGRATOR}

A PI controller with a washed out integrator is approximated using

$$
G_{c}(s)=K_{p}+K_{i} \frac{1}{s+1 / \tau_{h}}
$$


where $K_{p}=0.5, K_{i}=0.2$, and $\tau_{h}=3.2$. Figure 12 shows the root locus plot ( $K_{p}$ is varied with a zero fixed at $\left.-\left(\frac{1}{\tau_{h}}+\frac{K_{i}}{K_{p}}\right)=-0.7\right)$ and the loop gain frequency response with the controller in (21). When the controller
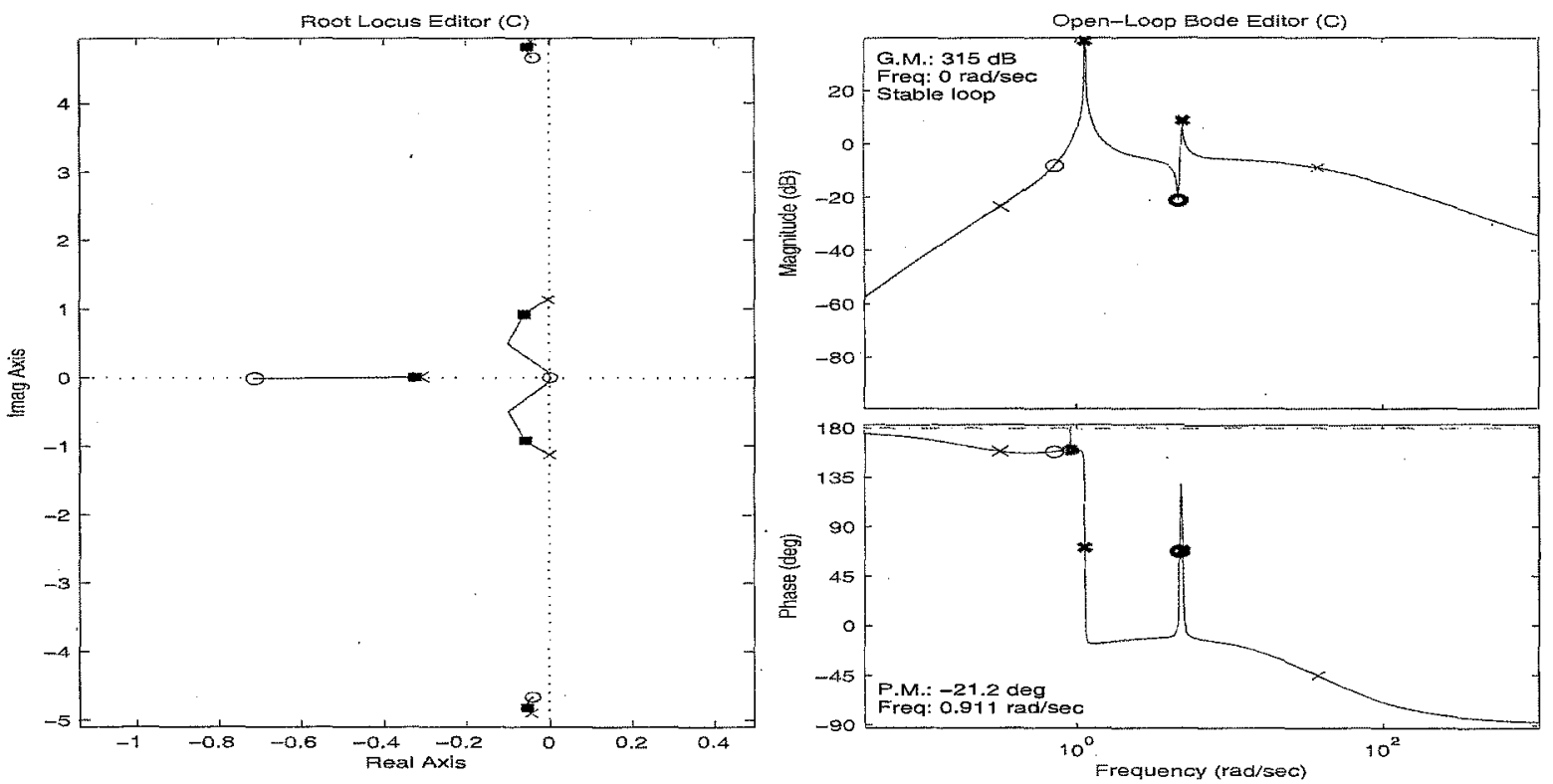

Figure 12: root locus and loop gain plots when the transfer function in (6), with $\zeta_{1}=0.003$, is regulated by the controller in (21)

in (21) was implemented in the experiment, the integrator still wound up quickly while this was not observed in simulation. This means that the instability is not caused by accelerometer bias. In Figure 13, the simulated acceleration responses of the nominal closed-loop system in which $\phi_{x_{i}}=\phi_{y_{i}}=0$ ("the plant model without coupling terms") are compared to those of the open-loop model in (5) in which $\phi_{x_{i}}, \phi_{y_{i}}$ are given by (19) ("OL"). More simulated accelerations are shown in Figure 14 for the cases where the PI controller is applied with and without the dead zone ("the plant model with dead zone" and "the plant model without dead zone"). While the $\mathrm{PI}$ controller reduces acceleration responses without nonlinearity, comparing Figure 13 and 14 (see "OL" and " the plant model with dead zone") shows that the PI controller does not overcome the dead zone and results in an acceleration response identical to that of the open-loop system. Moreover, Figure 14 shows that the controller is not effective in the absence of the dead zone in the actuator. That is, the controller applied to the model with the coupling terms $\phi_{x_{i}}$ and $\phi_{y_{i}}$ fails to effectively suppress vibrations whether the dead zone is present or not. This illustrates that the PI controller is not effective when the nonlinearities of displacements are present, implying that the presence of nonlinear coupling terms makes it extremely difficult to find a working PI controller.

\section{THE ROLE OF ADAPTATION}

Figure 15 shows acceleration responses when the controller in (21) is augmented by a NN with and without the dead zone nonlinearity. The control signal is activated at $20 \mathrm{sec}$. as in experimental results in Figure 8 . The adaptive signals compensate for both structural nonlinearities and the effect of dead zone. This is evidenced by the fact that there is little difference between the responses with and without the dead zone. Moreover, since the control architecture in Figure 3 does not involve a reference model, the NN augmented controllers produce nearly the same acceleration responses when augmenting the $\mathrm{P}$ and the $\mathrm{PI}$ controllers. This is shown in Figure 16. This is not surprising because the linear controllers, whether they are the $\mathrm{P}$ controller or the $\mathrm{PI}$ controllers, do not overcome the dead zone nonlinearities, and the control signals are dominated by the adaptive signals.

Finally, simulation results in which the model in (21) is regulated by the P controllers with NNs augmented are compared to the experimental data in which the structure is regulated by the same controller in Figure 17. Considering that the nonlinearities in the simulation model are either idealized or arbitrarily introduced, it is interesting that two results are in good agreement except those spikes caused by non-perfect synchronization in the thrusters. In essence, Figure 17 indicates that the NNs were effective in compensating for uncertainties that 


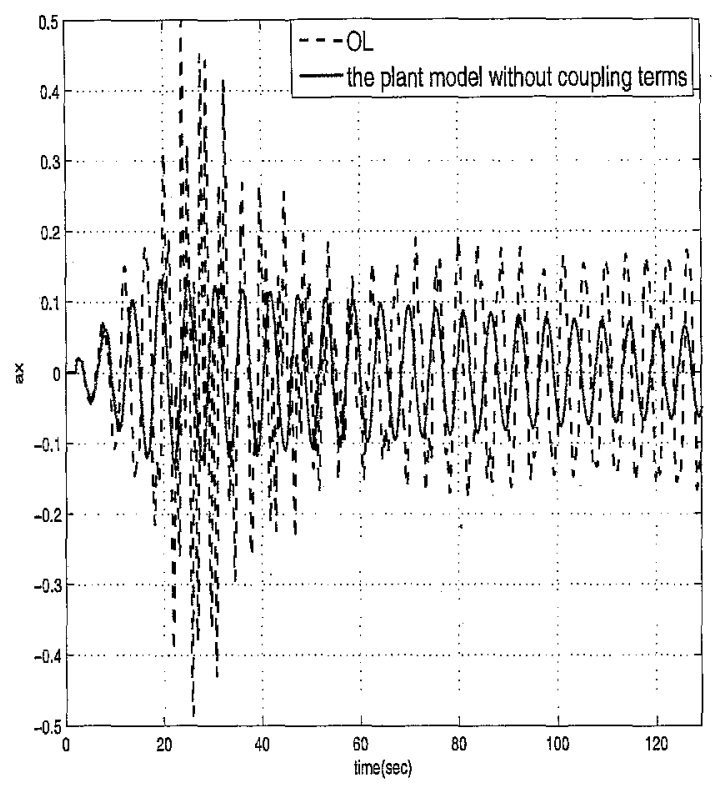

(a) $a_{x}$

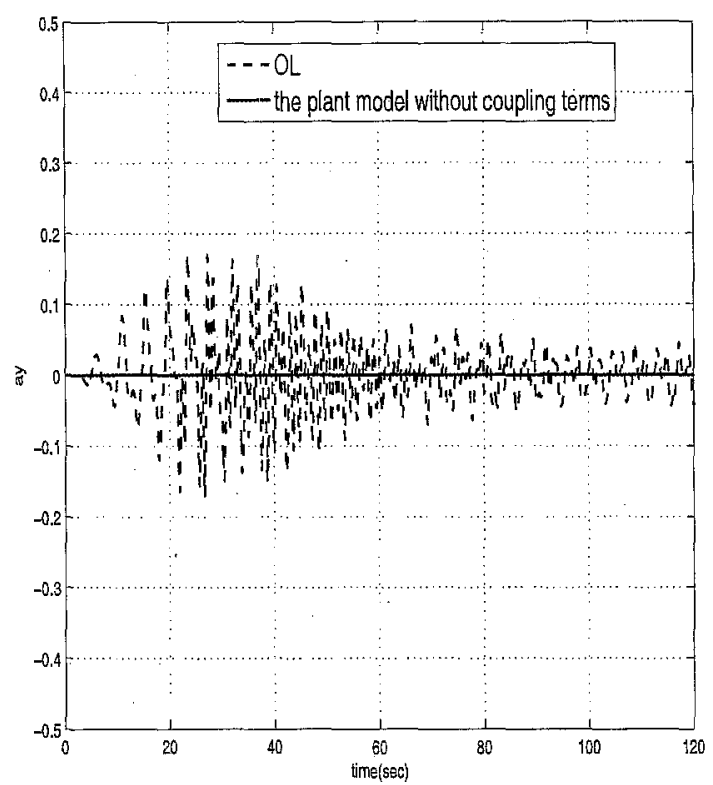

(b) $a_{y}$

Figure 13: Comparison of the open-loop responses to the plant model regulated by the controller in (21) in the absence of nonlinear terms

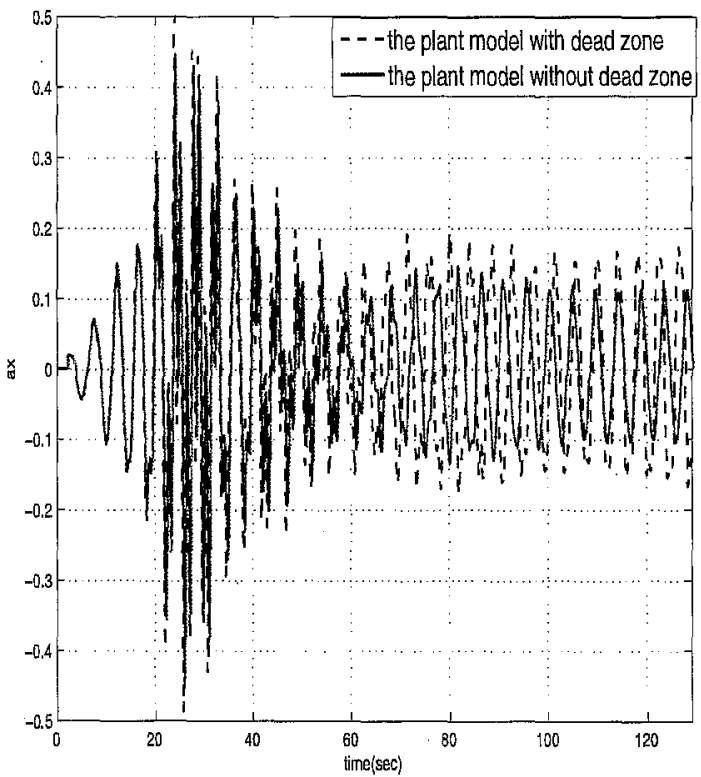

(a) $a_{x}$

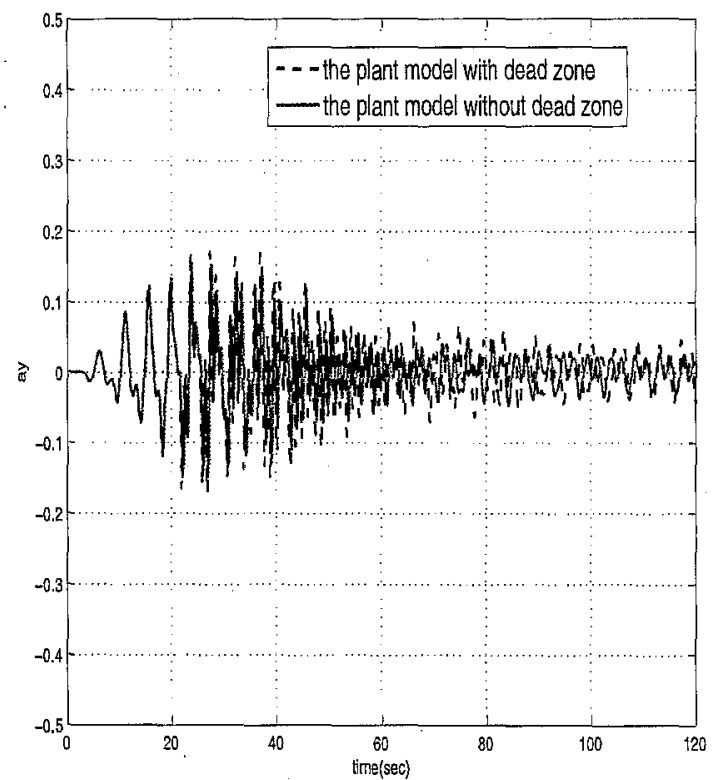

(b) $a_{y}$

Figure 14: Comparison of the closed-loop system regulated by the controller in (21) with and without the dead zone 


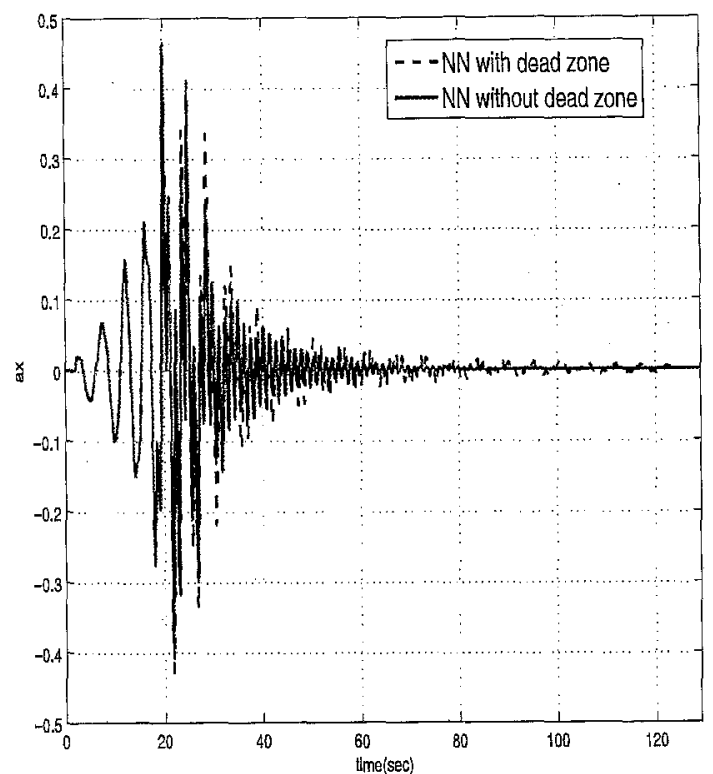

(a) $a_{x}$

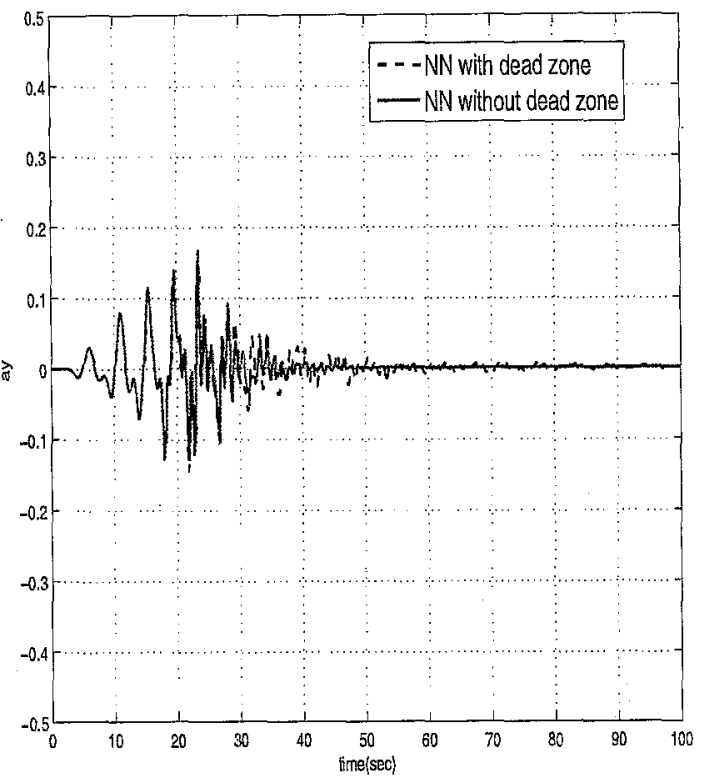

(b) $a_{y}$

Figure 15: Acceleration responses when the controller in (21) is augmented by a NN with and without dead zone

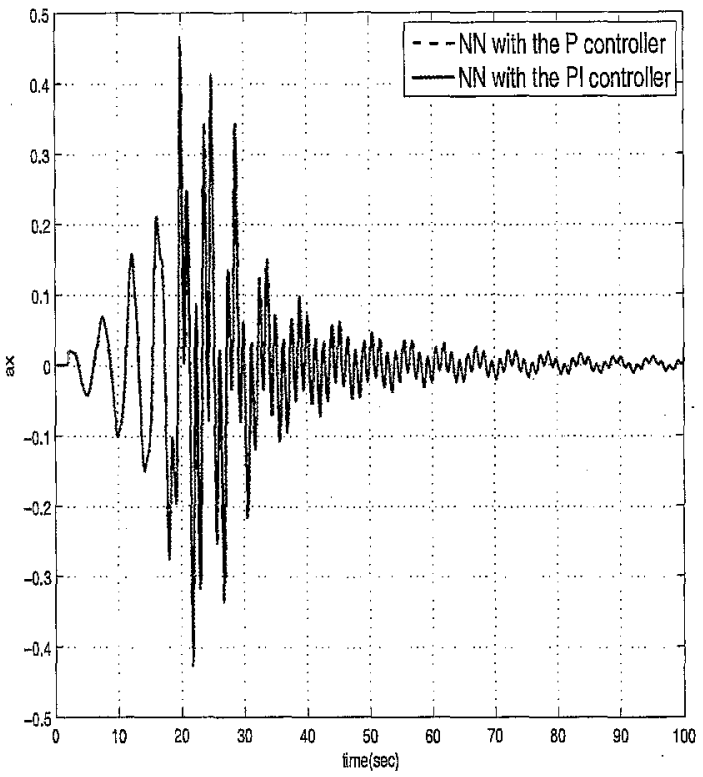

(a) $a_{x}$

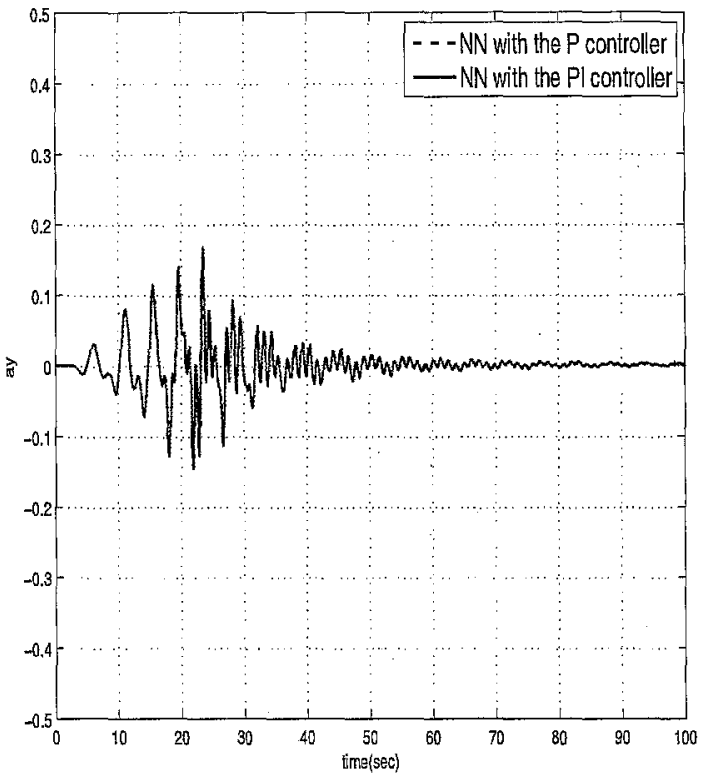

(b) $a_{y}$

Figure 16: Acceleration responses when the controller in (21) and the $\mathrm{P}$ controller are augmented by NNs 


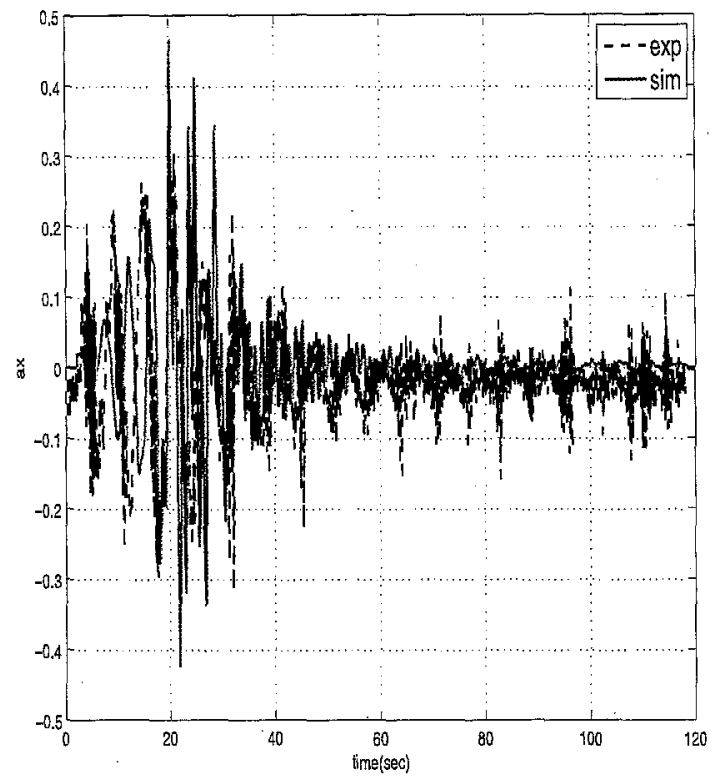

(a) $a_{x}$

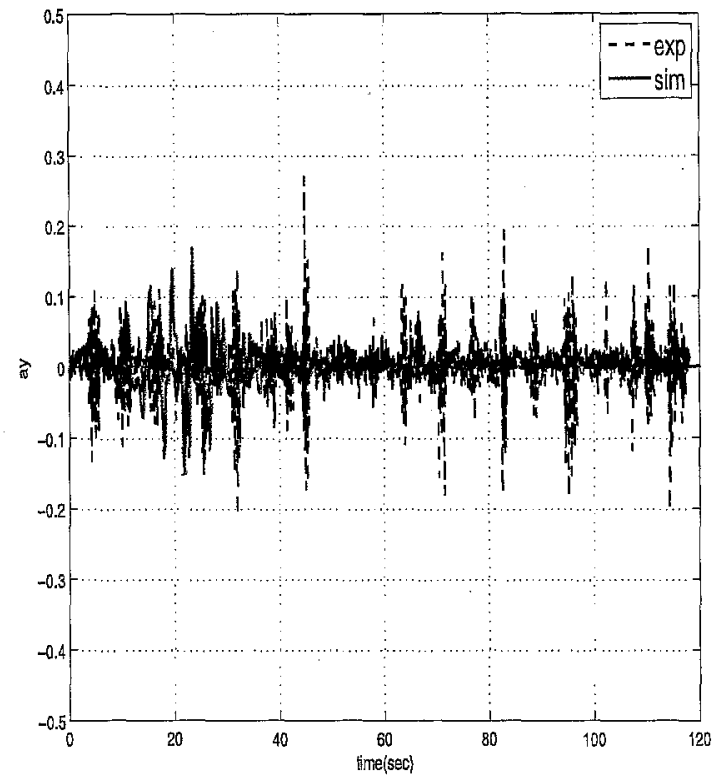

(b) $a_{y}$

Figure 17: Comparison of simulation and Experimental data when the $P$ controller is augmented by a NN

arise due to nonlinear actuation, couplings effects between the $X-Y$ dynamics, and unmodeled dynamics both in simulations and in the real implementation.

\section{FUTURE DIRECTIONS}

While the main results in this paper were obtained with the control architecture in Figure 3 , there are additional piezosensors, accelerometers, and piezoactuators that were not utilized. The main difficulty in utilizing these sensors and actuators was lack of modeling information. Figure 18 illustrates an manner in which NNs can be used to exploit the use of arrays of sensors and actuators. In model based control system design, exploitation of a large number of distributed sensors and actuators becomes a burdensome modeling task. One way a NN can be employed in the context of our present study is to use the same nominal controller for actuating the thrusters, but employ all the sensors in augmenting the nominal controller. This would extend to allowing for cross axis connections to the thrusters and to any other actuators that we may wish to employ. Each sensor output would influence the adaptive signal sent to each actuator in a different manner decided by the adaptation law. All that is required from a theoretical perspective is that the sign of the control effectiveness for each control effector be known, and that the relative degree of the regulated output be known with respect to the nominal control effectors. More generally, a NN-based adaptive controller can operate differently on each individual input of spatially distributed sensors, and the NN-outputs can be applied to each individual element in an array of spatially distributed actuators. The adaptive process can be used to decide on the weights of these interconnections. A theoretical basis for this development is proposed in [34].

\section{CONCLUSIONS}

We consider the feasibility of using neural network based adaptive control for a truss structure that may be used to support a solar sail membrane. An experimental study was performed using the SAFE (Solar Array Flight Experiment) boom located at NASA Marshall Space Flight Center. Whereas an attempt to design a proportionalintegral controller failed due to nonlinear actuation and the lack of a sufficiently accurate structural model, the adaptive controller was successful in attenuating structural response to disturbances. Simulation studies reveal that the design of decentralized linear controllers in the presence of structural nonlinearities/couplings is a difficult 


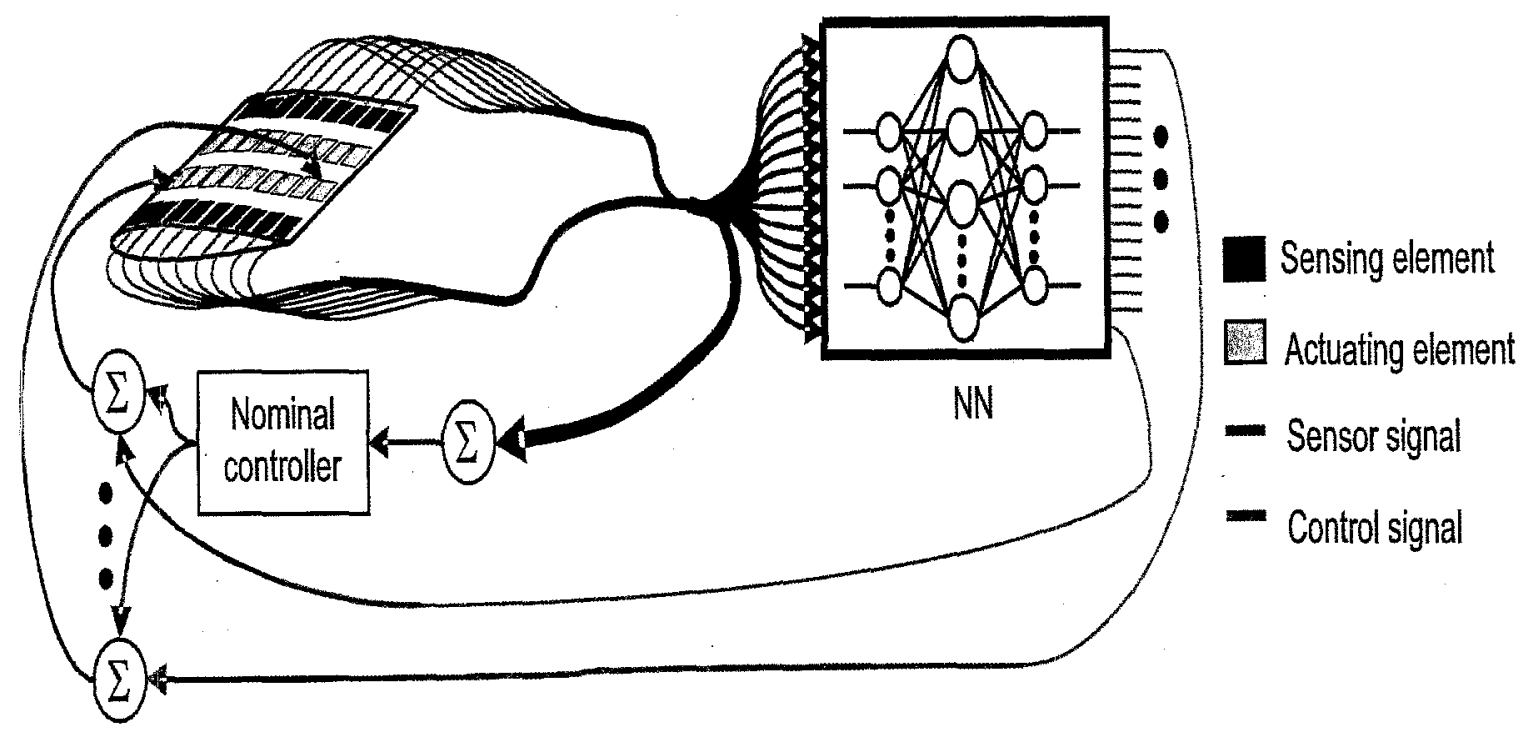

Figure 18: New control architecture

task. However these same controllers can be augmented with adaptive elements that render the closed loop system performance satisfactory. We also illustrate how neural network based adaptive control provides a natural architecture for making use of arrays of sensors and actuators, in which all sensor information is utilized and each actuator is individually compensated for the effects of nonlinearity and modeling error. Future research will be aimed at demonstrating this architecture as a means for fully utilizing the benefits afforded by distributed sensing and actuation.

\section{ACKNOWLEDGMENTS}

The authors would like to thank Mike Lau at NASA Marshall for integrating a Simulink-based controller with Labview. module, and Dr. Ali Kutay at Georgia Tech for providing the Figure 18 in which an array of sensors and actuators are utilized for controlling a wind-tunnel wing model, and Jerry Oakley at NASA Marshall for setting up the experiment, providing structural properties of the boom, and performing the main experimental tests presented in this paper. The first author would like to give special thanks to Jerry Oakley for helping him on a visit to NASA MSFC to perform experiments.

\section{REFERENCES}

[1] Mclnnes, C. R., Solar Sailing: Technology, Dynamics, and Mission Applications, Springer-Praxis Books in Astronautical Engineering, 2004.

[2] Murphy, D. and Wie, B., "Robust Thrust Control Authority for a Scalable Sailcraft," 14th AAS/AIAA Space Flight Mechanics Conference, AAS 04-285, Maui,HI, 2004.

[3] Wie, B., "Dynamic Modeling and Attitude Control of Solar Sail Spacecraft: Part 1," Proceedings of AIAA guidance, navigation and control conference, AIAA 2002-4572, Monterey, CA, 2002.

[4] Wie, B., "Dynamic Modeling and Attitude Control of Solar Sail Spacecraft: Part II," Proceedings of AIAA guidance, navigation and control conference, AIAA 2002-4573, Monterey, CA, 2002.

[5] Wie, B., "Solar Sail Attitude Control and Dynamics, Part 1," AIAA Journal of Guidance, Control \& Dynamics, Vol. 27, No. 4, 2004. 
[6] Rotunno, M., Basso, M., Pomè, A. B., and Sallusti, M., "A Comparison of Robust Attitude Control Techniques for a Solar Sail Spacecraft," Proceedings of AlAA guidance, navigation and control conference, AIAA 2005-6083, San Frrancisco, CA, 2005.

[7] Smith, S. W., Song, H., Baker, J. R., and Black, J., "Flexible Models for Solar Sail Control," 46th AIAA/ASME/ASCE/AHS/ASC Structures, Structural Dynamics and Materials Conference, AIAA 20051801, Austin, TX, 2005.

[8] Gorinevsky, D., Hyde, T., and Cabuz, C., "Distributed Localized Shape Control of Gossamer Space Structures," Proceedings of AIAA/ASME/ASCE/AHS/ASC Structures, Structural Dynamics, and Materials Conference, AIAA 2001-1197, Seattle, WA, 2001.

[9] Jha, A. K. and Inman, D. J., "Sliding Mode Control of a Gossamer Structure Using Smart Materials," Journal of Vibration and Control, Vol. 10, 2004, pp. 1199-1220.

[10] Ruggiero, E. J. and Inman, D. J., "Gossamer Spacecraft: Recent Trends in Design, Analysis, Experimentation, and Control," Journal of Spacecraft and Rockets, Vol. 43, No. 1, 2006, pp. 10-24.

[11] Sleight, D. W., Michii, Y., Lichodziejewski, D., and Derbès, B., "Structural Analysis of an Inflation-deployed Solar Sail with Experimental Validation," 41st AIAA/ASME/SAE/ASEE Joing Propulsion Conference, AIAA 2005-3727, Tucson, AZ, 2005.

[12] Hallauer, W., Lamberson, S., and Baer, C., "Active Vibration Damping of a Planar Truss Using Air-jet Thrusters," Experimental Mechanics, Vol. 31, No. 3, 1991, pp. 189-196.

[13] Casella, F., Locatelli, A., and Schiavoni, N., "Nonlinear controllers for vibration suppression in a large flexible structure," Control Engineering Practice, Vol. 4, No. 6, 1996, pp. 791-806.

[14] Allen, M., Bernelli-Zazzera, F., and Scattolini, R., "Sliding mode control of a large flexible space structure," Control Engineering Practice, Vol. 8, No. 8, 2000, pp. 861-871.

[15] Casella, F., Locatelli, A., and Schiavoni, N., "Modelling and control for vibration suppression in a large flexible structure with jet thrusters and piezoactuators," IEEE Transactions on Control Systems Technology, Vol. 10, No. 4; 2002, pp. 589-599.

[16] Adetona, O., Keel, L., Oakley, J., Kappus, K., Whorton, M., Kim, Y., and Rakoczy, J., "Model Correlation Study of a Retractable Boom for a Solar Sail Spacecraft," 53th JANNAF, Monterey, CA, Dec. 5-9 2005, pp. 2442-2447.

[17] Hovakimyan, N., Lavretsky, E., Yang, B.-J., and Calise, A., "Coordinated Decentralized Adaptive Output Feedback for Control of Interconnected Systems," Vol. 16, No. 1, 2005, pp. 185-194.

[18] Hornik, N., Stinchcombe, M., and White, H., "Multilayer feedforward networks are universal approximators," Neural Networks, Vol. 2, 1989, pp. 359-366.

[19] Ge, S., Lee, T., and Harris, C., Adaptive Neural Network Control of Robotic Manipulators, World Scientific, 1998.

[20] Lewis, F., Jagannathan, S., and Yeșildirek, A., Neural Network Control of Robot Manipulators and Nonlinear Systems, Taylor \& Francis, 1999.

[21] Spooner, J. T., Maggiore, M., Ordóñez, R., and Passino, K. M., Stable Adaptive Control and Estimation for Nonlinear Systems- Neural and Fuzzy Approximator Techniques, John Wiley \& Sons, New York, NY, 2002.

[22] Lavretsky, E., Hovakimyan, N., and Calise, A., "Upper Bounds for Approximation of Continuous-Time Dynamics Using Delayed Outputs and Feedforward Neural Networks," IEEE Transactions on Automatic Control, Vol. 48, No. 9, 2003, pp. 1606-1610.

[23] Kim, N., Calise, A. J,, Hovakimyan, N., Prasad, J., and Corban, J. E., "Adaptive Output Feedback for High-Bandwidth Flight Control," AIAA Journal of Guidance, Control \& Dynamics, Vol. 25, No. 6, 2002, pp. 993-1002. 
[24] Calise, A., Yang, B.-J., and Craig, J., "An Augmenting Adaptive Approach to Control of Flexible Systems," AIAA Journal of Guidance, Control \& Dynamics, Vol. 27, No. 3, 2004, pp. 387-396.

[25] Yang, B.-J., Hovakimyan, N., Calise, A., and Craig, J., "Experimental Validation of an Augmenting Approach to Adaptive Control of Uncertain Nonlinear Systems," Proceedings of AlAA guidance, navigation and control conference, AIAA-2003-5715, Austin, TX, 2003, (Submitted to IEEE Transactions on Control System Technology, 2006).

[26] Yang, B.-J., Calise, A., and Craig, J., "Adaptive Output Feedback Control of a Flexible Base Manipulator," Proceedings of AIAA guidance, navigation and control conference, AIAA-2004-5322, Providence, RI, August 2004, (Accepted for publication in AlAA Journal of Guidance, Control \& Dynamics, 2007).

[27] Yang, B.-J., Calise, A., and Craig, J., "Adaptive Output Feedback Control of a Flexible Base Manipulator," AIAA Journal of Guidance, Control \& Dynamics, 2007, Accepted for publication.

[28] Kutay, A. T., Fowler, J. M., Calise, A. J., and D'Andrea, R., "Distributed Adaptive Output Feedback Control Design and Application to a Formation Flight Experiment," Proceedings of AIAA guidance, navigation and control conference, Aug. 2005.

[29] Hovakimyan, N., Yáng, B.-J., and Calise, A. J., "Adaptive Output Feedback Control Methodology Applicable to Non-Minimum Phase Nonlinear Systems," Automatica, Vol. 42, No. 4, April 2006, pp. 513-522.

[30] Whorton, M. S., High Performance, Robust Control of Flexible Space Structures, Ph.D. thesis, Georgia Institute of Technology, School of Aerospace Engineering, Atlanta, GA 30332, aug 1997.

[31] Analog Devices, Inc., www.analog.com, Small and Thin \pm 5 g Accelerometer-ADXL320, rev.0 ed., 2004.

[32] Clippard Instrument Laboratory, Inc., www.clippard.com, EVP Series Proportional Control Valves, Oct. 2003.

[33] Balas, M. J., "Direct Velocity Feedback Control of Large Space Structures," AIAA Journal of Guidance, Control \& Dynamics, Vol. 2, No. 3, May-June 1979, pp. 252-253.

[34] Calise, A., Yang, B.-J., Craig, J., and Kim, K., "Hierarchical Centralized Adaptive Control of a Flexible Complex System," Proceedings of AIAA guidance, navigation and control conference, Hilton Head Island,SC, Aug. 2007, Accepted for presentation. 